\title{
Preexcitation Syndrome with a Mahaim-type Accessory Pathway
}

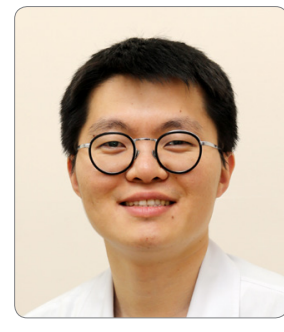

Kwang No Lee
Kwang-No Lee, MD; Young-Hoon Kim, MD, PhD

Division of Cardiology, Department of Internal Medicine, Korea University Medical Center, Seoul, Republic of Korea

Received: August 28, 2017

Accepted: September 28, 2017

Correspondence: Young-Hoon Kim, MD, PhD

Division of Cardiology, Department of Internal

Medicine, Korea University Medical Center, 73

Inchon-ro, Seongbuk-gu, Seoul 02841, Republic

of Korea.

Tel: +82-2-920-5445, Fax: +82-2-920-6395

Email:yhkmd@unitel.co.kr

Copyright (C) 2017 The Official Journal of Korean Heart Rhythm Society Editorial Board \& MMK Co., Ltd.

\begin{abstract}
Wide QRS complex tachycardia with a left bundle branch block pattern can be caused by supraventricular tachycardia with aberrant conduction, preexcitation syndrome mediated through a right-sided accessory pathway, and/or ventricular tachycardia. The use of atrial pacing maneuvers can be beneficial for unmasking minimal preexcitation to differentiate between these conditions. Here, we report a case of successful radiofrequency catheter ablation of a Mahaim fiber in a patient with wide QRS complex tachycardia.
\end{abstract}

Key Words: - Mahaim Fiber -Wide QRS complex Tachycardia - Left Bundle Branch Block - Preexcitation Syndrome

\section{Introduction}

Mahaim fibers were first described as accessory connections arising from the atrioventricular node (AVN), from the Hisbundle, or from fascicles to the right ventricle (RV) by Ivan Mahaim in $1938,{ }^{1}$ and have since been recognized as atriofascicular, nodofascicular, nodoventricular, and fasciculoventricular bypass tracts with antegrade decremental properties. ${ }^{2,3}$ Here, we present a case of wide QRS complex tachycardia (WQRST) involving the atriofascicular bypass tract in the right atrium (RA) that was initially misdiagnosed as atypical atrioventricular nodal reentrant tachycardia (AVNRT) at another hospital and that recurred following the initial procedure.

\section{Case}

A 45-year-old female presented with suspected recurrent WQRST after initial catheter ablation. She had undergone an electrophysiologic study (EPS) for intermittent palpitations with documented tachycardia two years prior (Figure 1A). On initial EPS, dual AVN physiology with an AH jump was demonstrated. The concentric retrograde atrial activation sequence was not advanced by His-refractory premature ventricular extrastimulus during tachycardia. The VA interval during tachycardia was 130 ms. However, tachycardia was still induced despite several attempts of slow pathway ablation including within the coronary sinus. Tachycardia frequently recurred starting three days after the procedure. 
A baseline 12-lead electrocardiogram (ECG) revealed normal sinus rhythm with a PR interval of $118 \mathrm{~ms}$ and a QRS duration of $90 \mathrm{~ms}$ (Figure 1B). EPS was performed using a duodecapolar catheter (Livewire $^{\text {txx }}$; St. Jude Medical, Minneapolis, MN, USA) in the RA, a decapolar catheter in the coronary sinus, and two quadripolar catheters in the right ventricle (RV) and the Hisbundle region. Atrial pacing augmented the degree of preexcitation with His potential followed by the potential of the RV apex. The degree of preexcitation over an accessory pathway was changed with different atrial pacing cycle lengths. Preexcitation was augmented at faster pacing, which led to prolongation of both the AH interval and stimulus-to-RV interval with reversal of activation sequences of the His and RV potentials (Figure 2). Adenosine blocked antegrade conduction through the AVN but did not affect the bypass tact. WQRST was induced by ventricular programmed extra stimulation with a drive cycle length of $400 \mathrm{~ms}$ and a single extrastimulus interval of 250 ms. Tachycardia was terminated by a single ventricular extrastimulus. The earliest ventricular activation in the tricuspid annulus was mapped by atrial pacing and was ablated with Mahaim potential (Figure 3). Preexcitation disappeared in three seconds, along with the transition of the ventricular activation sequence in the tricuspid annulus.

On post-ablation EPS, tachycardia was not induced. There was
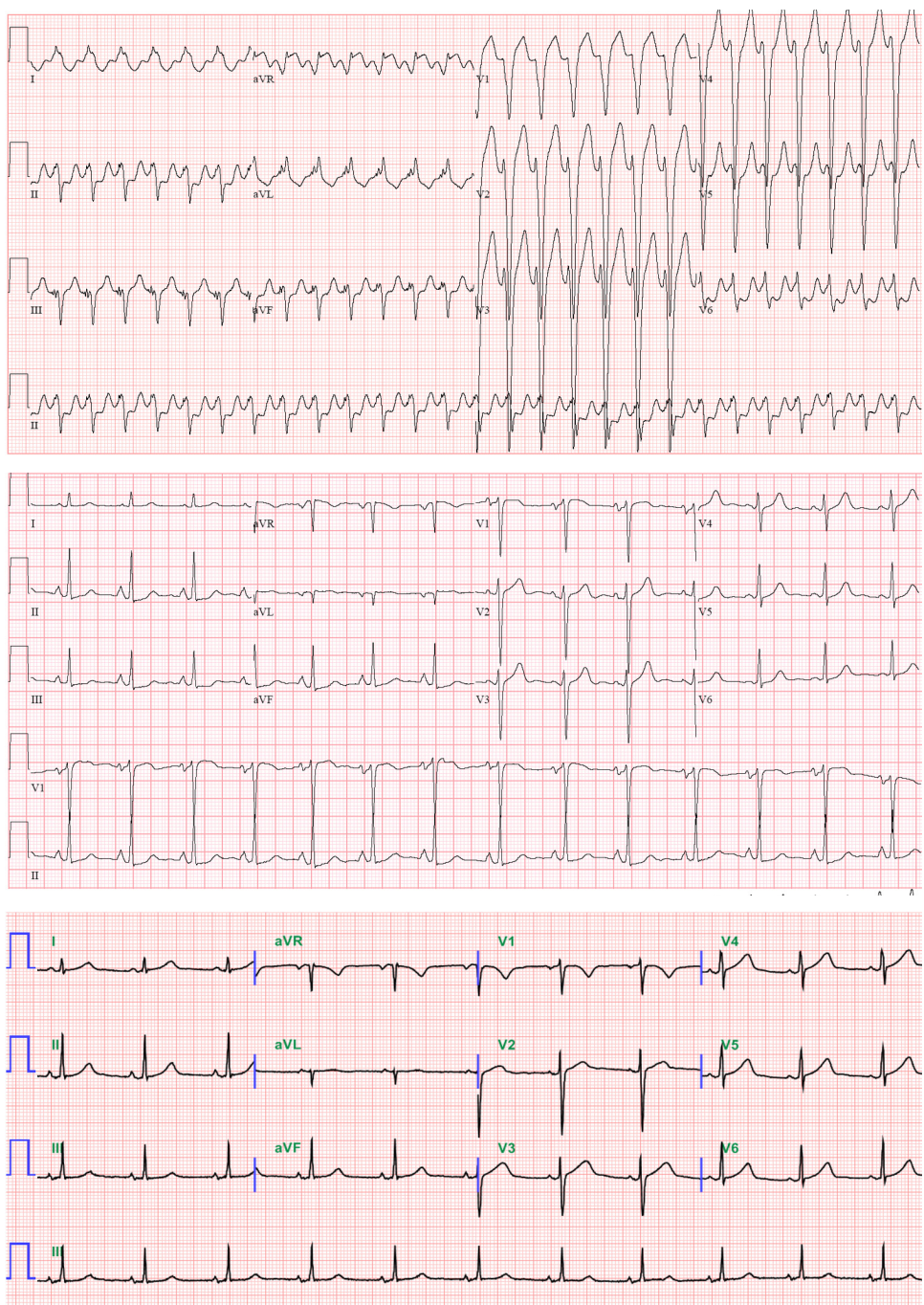

Figure 1. Surface 12-lead ECGs of sinus rhythm with WQRST (A), inapparent preexcitation before the ablation (B), and sinus rhythm after the ablation (C) 

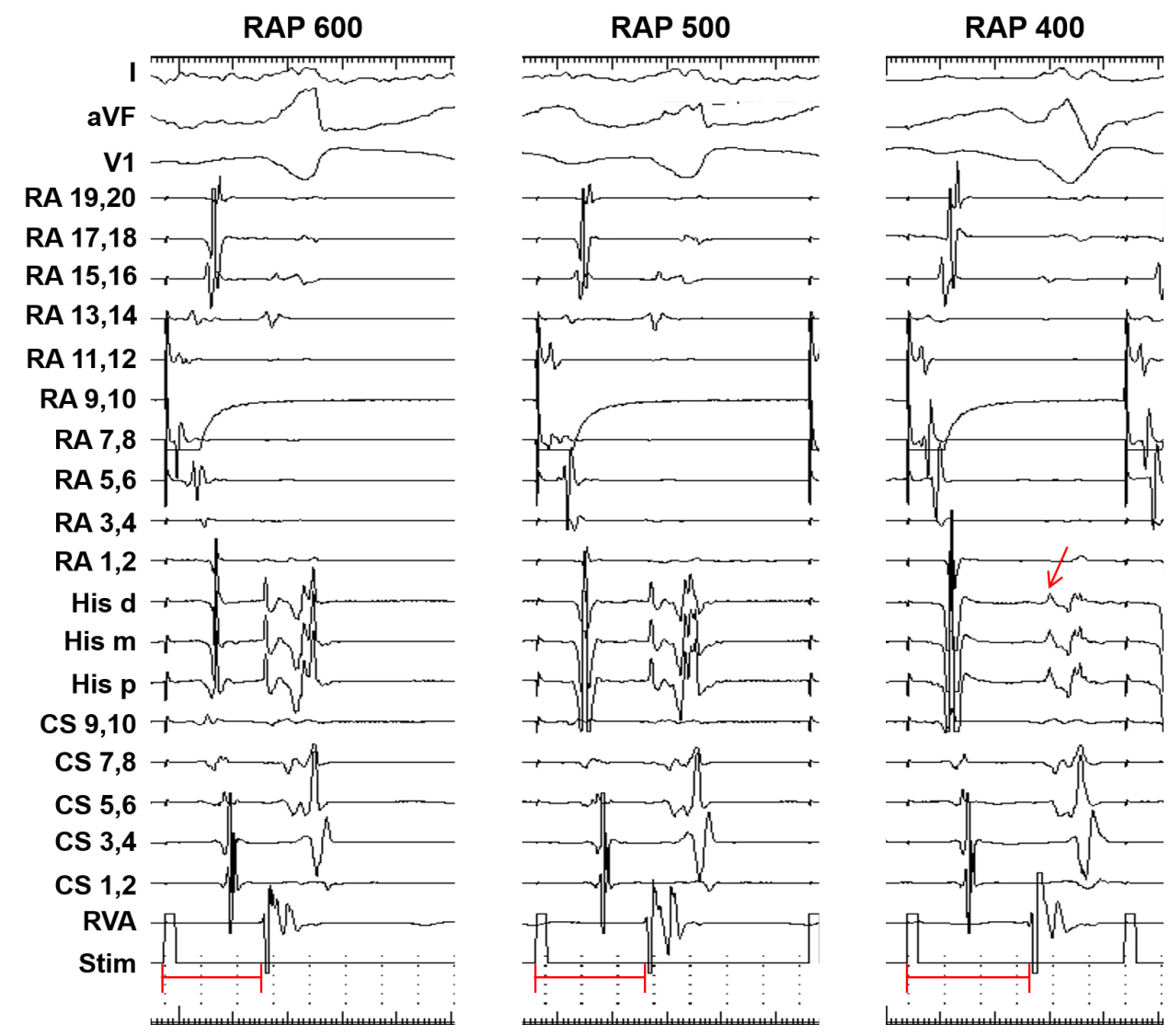

Figure 2. Changes in the degree of preexcitation over accessory pathway with atrial pacing. During atrial pacing at a slow cycle length (600 ms and $500 \mathrm{~ms}$ ), fusion is noted between ventricular activation over the accessory pathway and the AVN-His system. Augmented preexcitation is seen with faster atrial pacing $(400 \mathrm{~ms})$ that leads to more prolonged $\mathrm{A}$ to $\mathrm{H}$ potential than stimulus to the RVA, and the reversal of the AH to RVA sequence.

AH, atrium to his; AVN, atrioventricular node; CS, coronary sinus; His, His bundle; RA, right atrium; RAP, rapid atrial pacing; RVA, right ventricular apex; Stim, stimulus
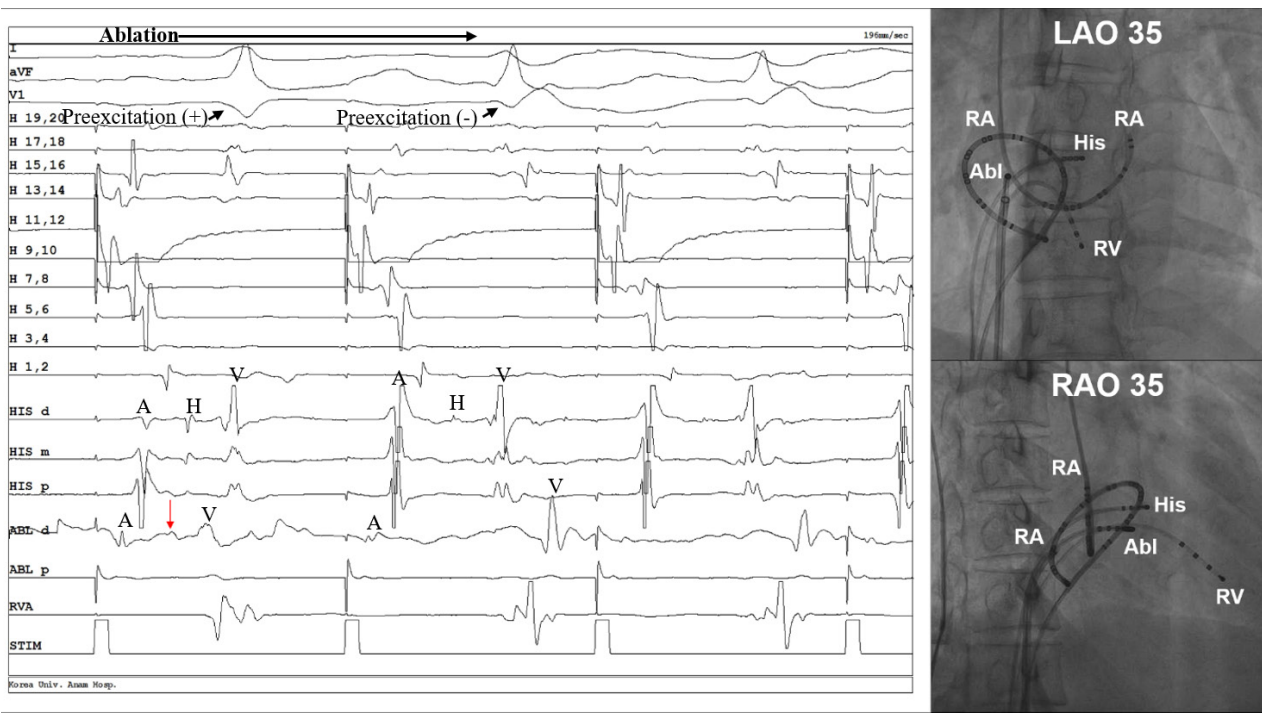

Figure 3. Mahaim potential (arrow) at the right lateral free wall on the tricuspid annulus and the modification of QRS complex morphology during Mahaim-type accessory pathway ablation. Preexcitation had been lost after the second beat. Fluoroscopic images on the left anterior oblique and right anterior oblique views show the position of the ablation catheter at the right atrial free wall on the tricuspid annulus.

A, atrium; Abl, catheter ablation; H, His bundle; His, His bundle; LAO, left anterior oblique; RA, right atrium; RAO; right anterior oblique, RV, right ventricle 
no antegrade conduction through the bypass tract on atrial pacing or adenosine injection. The patient remains free from symptoms 12 months after the procedure.

\section{Discussion}

Mahaim fibers were found to affect approximately $6 \%$ of accessory pathways in a previous study. ${ }^{4}$ Although the decremental antegrade conduction of these fibers is a unique electrophysiologic characteristic distinguishing them from other accessory pathways, there are several limitations to diagnosis. First, overt preexcitation is not usually seen in 12-lead ECGs of patients with an atriofascicular accessory pathway during sinus rhythm. Minimal preexcitation is supported by an $\mathrm{rS}$ pattern in lead III, absence of septal Q waves in leads I and V6, and terminal QRS slurring of notching. Second, although antidromic atrioventricular reentrant tachycardia (AVRT) over a Mahaim fiber typically has a left bundle branch block (LBBB) morphology, various QRS morphologies and axes may be observed, which can be difficult to differentiate from supraventricular tachycardia with LBBB aberrant conduction. ${ }^{5}$ Third, other heart conductions are associated with Mahaim fibers. Nearly $50 \%$ of patients with Mahaim fibers exhibit a dual AVN pathway or other accessory pathways. ${ }^{6-9}$ In cases such as these, the presence of dual AVN pathways may complicate the diagnosis of clinical tachycardia.

Progressive atrial pacing is helpful in identifying the presence of preexcitation in patients with WQRST. Rapid atrial pacing rate is expected to increase the degree of preexcitation via delayed conduction through the AVN, thereby promoting the contribution of accessory pathway conduction to ventricular excitation.

In conclusion, we report a right free wall accessory pathway with decremental antegrade conduction participating in antidromic AVRT, which was initially thought to be an instance of AVNRT. The diagnostic utility of atrial pacing for detecting inapparent preexcitation is highlighted.

\section{References}

1) Mahaim I, Benatt A. Nouvelles recherches sur les connexions supérieures de la branche gauche du faisceau de his-tawara avec la cloison interventriculaire. Cardiology (Switzerland). 1937;1:61-73.

2) Klein GJ, Guiraudon GM, Kerr CR, Sharma AD, Yee R, Szabo T, Wah JA. "Nodoventricular" accessory pathway: evidence for a distinct accessory atrioventricular pathway with atrioventricular node-like properties. J Am Coll Cardiol. 1988;11:1035-1040.

3) Tchou P, Lehmann MH, Jazayeri M, Akhtar M. Atriofascicular connection or a nodoventricular Mahaim fiber? Electrophysiologic elucidation of the pathway and associated reentrant circuit. Circulation. 1988;77:837-848.

4) Silva MA, Berardi G, Kraemer A, Nadalin E, Jorge JC. Catheter ablation of atriofascicular Mahaim fibers guided by the activation potential. Arq Bras Cardiol. 2003;80:66-70, 61-65.

5) Sternick EB, Cruz FE, Timmermans C, Sosa EA, Rodriguez LM, Gerken LM, Fagundes ML, Scanavacca MI, Wellens HJ. Electrocardiogram during tachycardia in patients with anterograde conduction over a Mahaim fiber: old criteria revisited. Heart Rhythm. 2004;1:406-413.

6) McClelland JH, Wang X, Beckman KJ, Hazlitt HA, Prior MI, Nakagawa H, Lazzara R, Jackman WM. Radiofrequency catheter ablation of right atriofascicular (Mahaim) accessory pathways guided by accessory pathway activation potentials. Circulation. 1994;89:2655-2666.

7) Haissaguerre M, Cauchemez B, Marcus F, Le Metayer P, Lauribe P, Poquet F, Gencel L, Clementy J. Characteristics of the ventricular insertion sites of accessory pathways with anterograde decremental conduction properties. Circulation. 1995;91:1077-1085.

8) Brugada J, Martinez-Sanchez J, Kuzmicic B, Figueiredo MO, Matas M, Pava LF, Navarro-Lopez F. Radiofrequency catheter ablation of atriofascicular accessory pathways guided by discrete electrical potentials recorded at the tricuspid annulus. Pacing Clin Electrophysiol. 1995;18:1388-1394.

9) Sternick EB, Gerken LM, Vrandecic M. Appraisal of "Mahaim" automatic tachycardia. J Cardiovasc Electrophysiol. 2002;13:244249. 